\title{
NONNEGATIVELY CURVED MANIFOLDS WITH SOULS OF CODIMENSION 2
}

\author{
GERARD WALSCHAP
}

J. Cheeger and D. Gromoll have classified the complete noncompact manifolds of nonnegative curvature in dimensions $\leq 3$ up to isometry (cf. [3]). This classification is partly based on the fact that for souls $S$ of dimension 1 (respectively codimension 1), the manifold $M$ is a locally isometrically trivial bundle over $S$ (respectively a flat line bundle over $S$ ).

In dimension 4 , an additional case may arise, namely $\operatorname{dim} S=\operatorname{codim} S=2$. This situation is analyzed in $\S 1$, where it is shown that when $S$ has codimension 2 , there is a Riemannian submersion $\pi: M \rightarrow S$, or else the normal bundle $\nu(S)$ of $S$ in $M$ is flat with respect to the induced connection. Those $M$ for which both conditions occur at the same time are the ones that split locally isometrically. Some results on total curvature follow. It turns out that the case where $\nu(S)$ is not flat is not as rigid as might be expected: in $\S 2$, the standard submersion metric on $S^{3} \times S^{1} \mathbf{R}^{2}$ is rather arbitrarily deformed while still retaining its nonnegative curvature. Finally, we show that given a metric of positive curvature on the $n$-sphere $S$, any 2-dimensional vector bundle over $S$ admits a metric of nonnegative curvature with soul isometric to $S$.

\section{Basic results}

$M$ will denote a complete noncompact manifold of nonnegative curvature with soul $S$. The reader is referred to [3] for the basic construction and main properties of souls, and to [6] for some facts about Riemannian submersions.

Lemma 1.1. Let $c:[0, a] \rightarrow S$ be a piecewise smooth curve joining $p$ and $q$ in $S$, and suppose $\gamma:[0, \infty) \rightarrow M$ is a ray originating at $p$. If $u \in M_{q}$ denotes the parallel translate of $\dot{\gamma}(0) \in M_{p}$ along $c$, then $t \mapsto \exp _{q}(t u)$ is a ray originating at $q$.

Proof. Since any piecewise smooth curve is a limit of broken geodesics, we may assume that $c$ is a geodesic, and thus extendable to $c: \mathbf{R} \rightarrow S$. Carry

Received December 23, 1986. 
out the basic soul construction at $p$, so that $M=\bigcup_{t \geq 0} C_{t}$, with $\gamma(t) \in \partial C_{t}$. Now $c(\mathbf{R})$ lies in the compact set $S$, and is therefore contained in some $C_{t_{0}}$, hence in every $C_{t}$ for $t \geq t_{0}$. By [3, Theorem 1.10], the distance function $s \mapsto d\left(c(s), \partial C_{t}\right)$ is concave. Being bounded from below and defined on all of $\mathbf{R}$, it must be constant. Consider the parallel field $X$ along $c$ with $X(0)=\dot{\gamma}(0)$, and set $c_{s}(t):=\exp _{c(s)} t X(s)$. Again by [3, Theorem 1.10], $c_{s}$ is a minimal geodesic from $c(s)$ to $\partial C_{t}$. Since this is true for all $t \geq t_{0}, c_{s}$ is a ray. q.e.d.

Recall that $M$ is diffeomorphic to the normal bundle $\nu(S)$ of $S$ in $M$. The following result was already known to D. Gromoll in the case $\operatorname{dim} M=4$.

Theorem 1.2. Suppose $\operatorname{codim} S=2$. Then one of the following holds:

(a) The normal bundle of $S$ is flat (with respect to the induced connection).

(b) There is a Riemannian submersion $\pi: M \rightarrow S$.

Remark. (a) and (b) are not mutually exclusive. In fact, their intersection consists precisely of those $M$ which are locally isometrically trivial bundles over $S$ (cf. 1.4).

Proof of 1.2. Since the fibers of $\nu$ are 2-dimensional, the reduced holonomy group $\Phi_{0}(p)$ of the connection is either trivial or isomorphic to $\operatorname{SO}(2) \cong$ $S^{1}$. The trivial case corresponds to (a). Assume then that $\Phi_{0}(p)$ is isomorphic to $S^{1}$ for each $p \in S$. The remaining part of the proof is divided into several steps. First, notice that every direction in the normal bundle yields a ray, i.e. given $v \in \nu(S),\|v\|=1, t \mapsto \exp (t v)$ is a ray. Indeed, since $M$ is noncompact, there is at least one ray emanating from any one point of $M$. Fix $p \in S$, and choose $v \in M_{p}$ so that $t \mapsto \exp _{p}(t v)$ is a ray. By [3, Theorem 5.1], $v \in \nu(S)$. Since $S$ is totally geodesic in $M$, a parallel section of $\nu$ along a curve will be parallel in $M$. By 1.1, $t \mapsto \exp (t u)$ is a ray for any $u$ in $\Phi_{0}(p) v$. Since $\Phi_{0}(p)$ is $S^{1}$, the result follows. Next, let $p \in S$, and carry out the basic soul construction at $p$. Then $S=C_{0}=\partial C_{0}$, and the closure of $B_{t}(S)$ equals $C_{t}$, where $B_{t}(S):=\{q \in M \mid d(q, S)<t\}$. To see this, consider a minimal connection $\gamma^{\prime}$ from a given $q \in M-S$ to $S$. Then $\gamma:=-\gamma^{\prime}$ is a ray with $\gamma\left(t_{0}\right)=q$, where $t_{0}:=d(q, S)$. Let $X$ denote the parallel vector field along some minimal geodesic $c:[0, a] \rightarrow S$ from $\gamma(0)$ to $p$, with $X(0)=\dot{\gamma}(0)$. Then $t \mapsto \tilde{\gamma}(t):=\exp t X(a)$ is a ray at $p, \tilde{\gamma}\left(t_{0}\right) \in \partial C_{t_{0}}$, and by $[3,1.10]$, $s \mapsto \exp _{c(s)} t_{0} X(s)$ is a curve in $\partial C_{t_{0}}$ from $q$ to $\tilde{\gamma}\left(t_{0}\right)$. In particular, $q \in \partial C_{t_{0}}$. Thus $\partial \bar{B}_{t_{0}}(S) \subset \partial C_{t_{0}}, t_{0}>0$. This also shows that $C_{0} \subset S$. Now assume $q$ is in $S$, and choose a minimal geodesic $c$ from $p$ to $q$. By the argument in $1.1, c(\mathbf{R})$ is contained in some $\partial C_{t}$. Then $p=c(0)$ belongs to $\partial C_{0} \cap \partial C_{t}$, so $t=0$. Hence $S \subset \partial C_{0}$. The inclusion $\partial C_{t} \subset \partial \overline{B_{t}}(S)$ now follows easily.

Finally, we show that $\exp _{\nu}: \nu(S) \rightarrow M$ is a diffeomorphism. Since every $q$ in $M$ has a minimal connection to $S$, $\exp _{\nu}$ is onto. Suppose there are two minimal connections $\gamma_{i}:\left[0, t_{0}\right] \rightarrow M$ from $S$ to $q, i=1,2$. This would 
contradict $\gamma_{1}\left(t_{0}+\delta\right) \in \partial C_{t_{0}+\delta}$, since the composite curve $\left.\left.\gamma_{2}\right|_{\left[0, t_{0}\right]} * \gamma_{1}\right|_{\left[t_{0}, t_{0}+\delta\right]}$ is a connection of length $t_{0}+\delta$ from $S$ to $\gamma_{1}\left(t_{0}+\delta\right)$ which can be shortened. Thus $\exp _{\nu}$ is $1-1$.

To complete the proof of 1.2 , recall that if $K$ denotes the connection map of $\nu(S)$, then

$$
\langle\langle a, b\rangle\rangle:=\langle K a, K b\rangle+\left\langle\pi_{\nu *} a, \pi_{\nu *} b\right\rangle, \quad a, b \in(T \nu)_{v}
$$

defines a metric on $\nu(S)$, called the connection metric, such that the projection $\pi_{\nu}: \nu(S) \rightarrow S$ becomes a Riemannian submersion.

Define $\pi:=\pi_{\nu} \circ \exp _{\nu}^{-1}: M \rightarrow S$. Then $\pi$ is a submersion, and to show $\pi$ is Riemannian, it suffices to establish the following:

(1) $\exp _{\nu *}$ maps the horizontal and vertical subspaces of $\pi_{\nu}$ onto mutually orthogonal subspaces.

(2) $\exp _{\nu *}$ is isometric on the horizontal subspaces.

So let $0 \neq z \in \nu(S), \pi_{\nu}(z)=: p, a \in(T \nu)_{z}$ horizontal, $b \in(T \nu)_{z}$ vertical. Since exp is radially isometric, we may assume $\left\langle\left\langle b, A_{z} z\right\rangle\right\rangle=0$, where $A_{z}: \nu_{p} \rightarrow\left(\nu_{p}\right)_{z}$ denotes the canonical isomorphism between the fiber through $p$ and its tangent space at $z$. Set $u:=\exp _{\nu *} b, w:=\exp _{\nu *} a$, and let $\gamma$ denote the ray $\gamma(t)=\exp (t z /\|z\|)$. $u$ determines a variation of $\gamma$ through rays emanating from $p$, and thus a Jacobi field $X$ along $\gamma$, with $X(0)=$ $0, X^{\prime}(0)=\left(A_{z}^{-1} b\right) /\|z\|$, and $X(\|z\|)=u$. Consider the geodesic $c: \mathbf{R} \rightarrow S$ with $\dot{c}(0)=\pi_{\nu_{*}} a=\pi_{*} w$. $c$ and $\gamma$ determine a flat totally geodesic rectangle $V(t, s)=\exp _{c(t)} s W(t)$, where $W$ is the parallel vector field along $c$ with $W(0)=z /\|z\|$. Thus the Jacobi field $Y$ along $\gamma, Y(s):=V_{*} \partial_{t \mid 0, s}$ is parallel along $\gamma$. Moreover, by uniqueness of horizontal lifts, $\|z\| \dot{W}(0)=a$, so that $w=\exp _{*} a=Y(\|z\|)$. Then $\|w\|=\|Y(\|z\|)\|=\|Y(0)\|=\left\|\pi_{\nu *} a\right\|=\|a\|$, which proves (2).

Finally, since $X$ and $Y$ are Jacobi and $Y$ is parallel, $\left\langle X^{\prime}, Y\right\rangle-\left\langle Y^{\prime}, X\right\rangle=$ $\left\langle X^{\prime}, Y\right\rangle$ is constant, and $\left\langle X^{\prime}, Y\right\rangle=\left.\left\langle X^{\prime}, Y\right\rangle\right|_{0}=\left\langle A_{z}^{-1} b, \pi_{\nu *} a\right\rangle /\|z\|=0$. Therefore, $\langle X, Y\rangle$ is constant, and $\langle u, w\rangle=\left.\langle X, Y\rangle\right|_{\|z\|}=\left.\langle X, Y\rangle\right|_{0}=0$, which proves (1). q.e.d.

We now examine the submersion case in more detail. For the sake of simplicity, $M$ and $S$ will be assumed oriented, even though this hypothesis is often unnecessary. In any case, local results carry through to nonorientable $M$, while similar global results can be obtained by considering the orientation covering.

Denote by $J$ the canonical complex structure on $\nu(S)$, i.e., $J U=V$ for (local) oriented orthonormal sections $\{U, V\}$ of $\nu$. Define vector fields $\tilde{\partial}_{r}, \tilde{\partial}_{\vartheta}$ on $\nu(S)-S$ as follows:

$$
\left.\tilde{\partial}_{r}\right|_{z}:=A_{z} z /\|z\|,\left.\quad \tilde{\partial}_{\vartheta}\right|_{z}:=A_{z} J z, \quad z \in \nu(S)-S,
$$


where $A$ is the isomorphism defined in 1.2. $\left(\tilde{\partial}_{r}, \tilde{\partial}_{\vartheta}\right.$, when restricted to a fiber, are just the standard polar coordinates vector fields.) Let $\partial_{r}$ and $\partial_{\vartheta}$ denote the corresponding $\exp _{\nu}$-related vector fields on $M-S$, with dual 1-forms $d r$ and $d \vartheta$. Observe that $\partial_{r}=\nabla d_{S}$, where $d_{S}$ is the distance function from the soul, while $\partial_{\vartheta}$, when restricted to a ray originating at $S$, is a Jacobi field $Y$ with initial conditions $Y(0)=0,\left\|Y^{\prime}(0)\right\|=1$. Moreover, $\left[\partial_{r}, \partial_{\vartheta}\right]=$ 0 , and if $\bar{X}$ is the horizontal lift of $X \in \mathfrak{X} S$, then $\left[\bar{X}, \partial_{r}\right]=\left[\bar{X}, \partial_{\vartheta}\right]=0$, since $\left[\tilde{X}, \tilde{\partial}_{r}\right]=\left[\tilde{X}, \tilde{\partial}_{\vartheta}\right]=0$ in $\nu(S)$, for the horizontal lift $\tilde{X}$ of $X$ to $\nu(S)$. Write $Z=Z^{h}+Z^{v}$ for the orthogonal splitting of $Z \in \mathfrak{X} M$ induced by the Riemannian submersion $\pi: M \rightarrow S$, with $Z^{v}$ tangent to the fiber.

Proposition 1.3. (i) Let $\Omega$ denote the curvature form of $\nu(S)$, viewed as a 2-form on $S$, i.e., $\Omega(X, Y):=\langle R(X, Y) U, J U\rangle$ for $X, Y \in \mathfrak{X} S, U \in \Gamma \nu$ of norm 1. If $\bar{X}, \bar{Y} \in \mathfrak{X} M$ are the horizontal lifts of $X, Y \in \mathfrak{X} S$, then

$$
[\bar{X}, \bar{Y}]^{v}=-\Omega(X, Y) \partial_{\vartheta}
$$

In particular, if the O'Neill tensor is zero (resp. nonzero) at some point $q$, then it is identically zero (resp. nowhere zero) on the fiber through $q$.

(ii) Set $G^{2}:=\left\langle\partial_{\vartheta}, \partial_{\vartheta}\right\rangle$, so that the fiber metric is $d r^{2}+G^{2} d \vartheta^{2}$. If the $O$ 'Neill tensor is nonzero on a fiber, then $G$ is bounded on that fiber. The intrinsic sectional curvature of a fiber equals the one induced by $M$,

$$
K_{\text {fiber }}=-G^{-1} G_{r r} \text {. }
$$

(iii) Consider $\nu(S)$ with the connection metric, and replace the standard flat fiber metric $d r^{2}+r^{2} d \vartheta^{2}$ by $d r^{2}+\left(G \circ \exp _{\nu}\right)^{2} d \vartheta^{2}$. Then $\exp _{\nu}: \nu(S) \rightarrow M$ is an isometry.

Proof. As before, $\tilde{X}$ and $\bar{X}$ are the horizontal lifts of $X \in \mathfrak{X} S$ to $\nu(S)$ and $M$ respectively. Since $\exp _{\nu}$ preserves the orthogonal splitting,

$$
\left.[\bar{X}, \bar{Y}]^{v}\right|_{\exp z}=\left.\exp _{*}[\tilde{X}, \tilde{Y}]^{v}\right|_{z}, z \in \nu(S) .
$$

If $R$ and $K$ denote the curvature tensor and the connection map of $\nu(S)$, then

$$
R(X, Y) z=-K[\tilde{X}, \tilde{Y}]_{z}
$$

or equivalently,

$$
\left.[\tilde{X}, \tilde{Y}]^{v}\right|_{z}=-A_{z} R(X, Y) z=-\Omega(X, Y) A_{z} J z=-\left.\Omega(X, Y) \tilde{\partial}_{\vartheta}\right|_{z}
$$

Applying $\exp _{\nu *}$ to the last equation now yields (i).

By O'Neill's formula and (i),

$$
\frac{3}{4} \Omega^{2}(X, Y) G^{2}=\frac{3}{4}\left\|[\bar{X}, \bar{Y}]^{v}\right\|^{2}=K_{X, Y}-K_{\bar{X}, \bar{Y}} \leq K_{X, Y},
$$

hence $G$ is bounded if $\Omega$ is nonzero. 
Consider a horizontal $u \in T M$. Since $\nabla_{u} \partial_{r}=0$, we have $l_{u}\left(\partial_{r}, \partial_{r}\right)=$ $l_{u}\left(\partial_{r}, \partial_{\vartheta}\right)=0$, where $l_{u}$ is the second fundamental form of the fiber with respect to $u$; the statement about the curvature of the fiber now follows from the Gauss equations. Finally, (iii) is implicitly contained in the proof of 1.2. q.e.d.

For any horizontal unit-speed geodesic $c: \mathbf{R} \rightarrow M, T:=\partial_{\vartheta} \circ c$ is a Jacobi field along $c$. Let $\mu(t)$ denote the principal curvature of the fiber through $c(t)$ with corresponding principal curvature direction $G^{-1} T$. Thus $S_{\dot{c}} \partial_{\vartheta}=\mu T(S$ is the second fundamental tensor of the fiber), and

$$
\mu=(G \circ c)^{-1}(G \circ c)^{\prime}=(G \circ c)^{-2}\left\langle T^{\prime}, T\right\rangle .
$$

Differentiating this equation yields:

$$
\mu^{\prime}=-K_{\dot{c}, T}+(G \circ c)^{-2}\left\|T^{\prime}\right\|^{2}-2 \mu^{2} .
$$

Suppose now that $\nu(S)$ is flat, or equivalently, that the O'Neill tensor is identically zero. Then $T^{\prime h}=0$, and since $T^{\prime v}=S_{\dot{c}} \partial_{\vartheta}$, we obtain

$$
\mu^{\prime}=-\mu^{2}-K_{\dot{c}, T}
$$

This in turn implies that $\mu \equiv 0$. For if $\varphi$ is an antiderivative of $\mu$, then

$$
\left(e^{\varphi}\right)^{\prime \prime}=e^{\varphi}\left(\mu^{\prime}+\mu^{2}\right) \leq 0 .
$$

Thus $e^{\varphi}$ is concave and bounded from below, hence constant, and $\mu \equiv 0$. Therefore, the fibers are totally geodesic. Together with the fact that $\nu$ is flat, this implies (cf. [9]):

Theorem 1.4. Assume $S$ has codimension 2. If $\nu(S)$ is flat and if every normal direction represents a ray, then $M$ is locally isometrically a product.

One should take care, when dealing with flat normal bundles, to distinguish them from trivial ones. Of course, if $S$ is topologically a 2-sphere, then $\nu(S)$ is trivial whenever it is flat. The converse is not true in general. Consider for example the free $\mathbf{R}$-action $\Gamma$ on $S^{2} \times \mathbf{R}^{2} \times \mathbf{R}$ given by $\left(q, u, t_{0}\right) \mapsto\left(\varphi_{t} q, e^{i t} u\right.$, $\left.t_{0}+t\right)$, where $\varphi_{t}$ denotes rotation by angle $t$ in $S^{2}$ about the $z$-axis, and $e^{i t}$ is rotation by angle $t$ in $\mathbf{R}^{2}$ around the origin. $\Gamma$ acts freely by isometries on the Riemannian product $S^{2} \times \mathbf{R}^{2} \times \mathbf{R}$, and there is a unique metric of nonnegative curvature on $M=S^{2} \times \mathbf{R}^{2} \times \mathbf{R} / \Gamma$ for which the projection $\rho: S^{2} \times \mathbf{R}^{2} \times \mathbf{R} \rightarrow M$ becomes a Riemannian submersion (cf. $\S 2$ ). $M$ is diffeomorphic to $S^{2} \times \mathrm{R}^{2}$, and under this identification, the soul $S$ turns out to be $S^{2} \times 0$, while the submersion $\pi: M \rightarrow S$ becomes the projection $\pi_{1}: S^{2} \times \mathbf{R}^{2} \rightarrow S^{2} \times 0$. Nevertheless, the metric on $M$ is not a Riemannian product, hence the normal bundle of $S$ is not flat even though it is trivial. The key obstruction here is that the fibers are not totally geodesic, as one can easily check. Indeed, one has 
Theorem 1.5. If $M^{4}$ is a trivial bundle over $S$, and $\pi: M \rightarrow S$ has totally geodesic fibers, then $\pi$ is a locally isometrically trivial fibration.

Together with 1.4, this result immediately implies

Corollary 1.6. Suppose $M^{4}$ has soul $S$ diffeomorphic to a 2-sphere, and every direction in $\nu(S)$ is a ray direction. Then the following statements are equivalent:

(i) $\nu(S)$ is flat.

(ii) $M$ is diffeomorphic to $S \times \mathbf{R}^{2}$ and $\pi: M \rightarrow S$ has totally geodesic fibers.

(iii) $M=S \times P_{2}$ isometrically, where $P_{2}$ is $\mathbf{R}^{2}$ together with some metric of nonnegative curvature.

To prove 1.5, we need

\section{Lemma 1.7.} $M$.

(i) $\operatorname{div} \partial_{\vartheta}=\partial_{\vartheta} \ln G$. If $\partial_{\vartheta}$ is divergence-free, then it is a Killing field on

(ii) If $\nu(S)$ is not flat and $\pi: M \rightarrow S$ has totally geodesic fibers, then $\partial_{\vartheta}$ is a Killing field.

Proof of 1.7. If $\left\{X_{i}\right\}$ is a local orthonormal basis of basic vectors fields, then

$$
\begin{aligned}
\operatorname{div} \partial_{\vartheta} & =G^{-2}\left\langle\nabla_{\partial_{\vartheta}} \partial_{\vartheta}, \partial_{\vartheta}\right\rangle+\left\langle\nabla_{\partial_{r}} \partial_{\vartheta}, \partial_{r}\right\rangle+\sum_{i}\left\langle\nabla_{X_{i}} \partial_{\vartheta}, X_{i}\right\rangle \\
& =\partial_{\vartheta} \ln G-\left\langle\partial_{\vartheta}, \nabla_{\partial_{r}} \partial_{r}\right\rangle-\sum_{i}\left\langle\partial_{\vartheta},\left(\nabla_{X_{i}} X_{i}\right)^{v}\right\rangle \\
& =\partial_{\vartheta} \ln G .
\end{aligned}
$$

Assume $\operatorname{div} \partial_{\vartheta}=0$. Then

$$
\begin{gathered}
\left\langle\nabla_{X_{i}} \partial_{\vartheta}, X_{j}\right\rangle+\left\langle\nabla_{X_{j}} \partial_{\vartheta}, X_{i}\right\rangle=-\left\langle\partial_{\vartheta},\left(\nabla_{X_{i}} X_{j}\right)^{v}+\left(\nabla_{X_{j}} X_{i}\right)^{v}\right\rangle=0, \\
\left\langle\nabla_{X_{i}} \partial_{\vartheta}, \partial_{\vartheta}\right\rangle+\left\langle\nabla_{\partial_{\vartheta}} \partial_{\vartheta}, X_{i}\right\rangle=\left\langle\left[X_{i}, \partial_{\vartheta}\right], \partial_{\vartheta}\right\rangle=0, \\
\left\langle\nabla_{X_{i}} \partial_{\vartheta}, \partial_{r}\right\rangle+\left\langle\nabla_{\partial_{r}} \partial_{\vartheta}, X_{i}\right\rangle=-\left\langle\partial_{\vartheta}, \nabla_{X_{i}} \partial_{r}+\nabla_{\partial_{r}} X_{i}\right\rangle=0 \\
\left\langle\nabla_{\partial_{\vartheta}} \partial_{\vartheta}, \partial_{r}\right\rangle+\left\langle\nabla_{\partial_{r}} \partial_{\vartheta}, \partial_{\vartheta}\right\rangle=\left\langle\left[\partial_{r}, \partial_{\vartheta}\right], \partial_{\vartheta}\right\rangle=0 .
\end{gathered}
$$

Thus $\partial_{\vartheta}$ is a Killing field. To prove (ii), choose $p \in S$ so that $\Omega_{p} \neq 0$. Since the fibers are totally geodesic, $[\bar{X}, \bar{Y}]^{v}=-\Omega(X, Y) \partial_{\vartheta}$ is Killing on the fiber through $p$, implying $\partial_{\vartheta} G=0$ on this fiber. But for any basic $X, X \partial_{\vartheta} G=\partial_{\vartheta} X G=0$, so that $\partial_{\vartheta} G \equiv 0$ on $M$. By (i), $\partial_{\vartheta}$ is Killing on $M$.

Proof of 1.5. If $\pi$ is not locally isometrically trivial, then $\nu(S)$ cannot be flat by 1.4. By 1.7, $\partial_{\vartheta}$ is a Killing field. Fix some positive $r$, and consider the set $N$ of points of $M$ at distance $r$ from $S . N$ has nonnegative curvature by the Gauss equations, is diffeomorphic to $S \times S^{\mathbf{1}}$, and thus admits a parallel vector field $Z$ by basic harmonic theory or [3]. Then $\left\langle Z, \partial_{\vartheta}\right\rangle$ is constant, and 
since $G=\left\|\partial_{\vartheta}\right\|$ is also constant on $N$, the same must be true for the angle between $Z$ and $\partial_{\vartheta}$. Choose $p \in S$ so that $\Omega_{p} \neq 0$, and let $q \in N \cap \pi^{-1}(p)$. If $\bar{X}, \bar{Y}$ are basic orthonormal, equation (2.2) in $\S 2$ yields:

$$
\nabla_{\bar{X}_{q}} \partial_{\vartheta}=\frac{1}{2} \Omega_{p}(X, Y) G^{2} \bar{Y}_{q} \neq 0, \quad \nabla_{\bar{Y}_{q}} \partial_{\vartheta}=-\frac{1}{2} \Omega_{p}(X, Y) G^{2} \bar{X}_{q} \neq 0 .
$$

But $0=\bar{X}\left\langle Z, \partial_{\vartheta}\right\rangle=\frac{1}{2} \Omega(X, Y) G^{2}\langle\bar{Y}, Z\rangle$, so that $Z \perp \bar{Y}$ on the fiber over $p$. Similarly $Z \perp \bar{X}$, and $Z$ is then vertical on this fiber. Hence $Z$ is vertical everywhere, and so $\partial_{\vartheta}$, being a constant multiple of $Z$, is a parallel vector field, contradicting $\nabla_{\bar{X}_{q}} \partial_{\vartheta} \neq 0$. Thus $\pi$ is locally isometrically trivial. q.e.d.

Recall that the total curvature of an oriented complete even-dimensional manifold $M$ is defined as $\int_{M} \chi$ (if it exists), where $\chi$ is the Chern-Euler form of $M$. When $\operatorname{dim} M=2, \chi=(1 / 2 \pi) K$ ( $K$ is the sectional curvature), and for $K \geq 0$, it is known that the total curvature is bounded between 0 and 1 (cf. [4]).

Lemma 1.8. Suppose $\Omega \neq 0$ at some $p \in S$. Then the fiber through $p$ has total curvature 1. In particular, if $\pi: M \rightarrow S$ has totally geodesic fibers and is not locally a Riemannian product, then every fiber has total curvature 1.

Proof. By 1.3(ii), $G$ is bounded on the fiber through $p$. Since $r \mapsto G(r, \vartheta)$ is concave and positive, $G_{r} \rightarrow 0$ as $r \rightarrow \infty$. Thus

$$
\int_{0}^{\infty}-G_{r r \mid r, \vartheta} d r=\lim _{r \rightarrow 0} G_{\left.r\right|_{r, \vartheta}}=1,
$$

and the total curvature of the fiber through $p$ is:

$$
\frac{1}{2 \pi} \int_{\text {fiber }} K_{\text {fiber }}=\frac{1}{2 \pi} \int_{0}^{2 \pi} \int_{0}^{\infty}-G_{r r} d r d \vartheta=1, \quad \text { by } 1.3(\mathrm{ii}) .
$$

If $M$ is not locally isometrically a product, then $\Omega$ is nonzero at some $p \in S$ by 1.4. Thus the fiber through $p$ has total curvature 1 . Since the fibers are totally geodesic, they are all isometric to one another (cf. [5]), and the statement follows. q.e.d.

It is known that the total curvature of any 4-dimensional oriented manifold of nonnegative curvature exists, and is bounded between 0 and the Euler characteristic of $M$ (cf. [7]). Assume $\operatorname{dim} M=4$. Under our additional assumptions, namely $\operatorname{dim} S=2$ and every normal direction represents a ray, we can prove a stronger result:

Theorem 1.9. Let $\kappa(p)$ denote the total curvature of the fiber through $p \in S, \kappa: S \rightarrow[0,1]$. Then the total curvature of $M^{4}$ equals

$$
\frac{1}{2 \pi} \int_{S} \kappa K_{S}
$$

where $K_{S}$ is the sectional curvature of $S$. 
Assume furthermore that $S$ is diffeomorphic to the 2-sphere (the only other possibility is $S=$ flat torus, in which case the total curvature of $M$ is 0 ), and that $\pi: M \rightarrow S$ has totally geodesic fibers. Then the total curvature of $M$ is 2 , unless $M=S \times P_{2}$ isometrically, in which case it is $2 \kappa$.

Proof. Let $M^{r}:=\{q \in M \mid d(q, S) \leq r\}$. Thus each $\partial M^{r}$ is diffeomorphic via $\exp _{\nu}^{-1}$ to the sphere bundle of radius $r$ over $S$, and admits the restriction of $\nabla d_{S}=\partial_{r}$ as unit normal vector field. $\omega_{r}$ and $\omega_{s}$ will denote the volume forms of $\partial M^{r}$ and $S$ respectively. The Gauss-Bonnet theorem for manifolds with boundary then yields:

$$
\int_{M^{r}} \chi=\chi(S)+\int_{\partial M^{r}} g_{r} \omega_{r}
$$

where $\chi(S)$ is the Euler characteristic of $S$, and

$$
g_{r}(q)=\left(-1 / 4 \pi^{2}\right)\left\{\lambda_{1} K_{23}+\lambda_{2} K_{13}+\lambda_{3} K_{12}+\lambda_{1} \lambda_{2} \lambda_{3}\right\},
$$

(cf. [7]). Here the $\lambda_{i}$ are the principal curvatures of $\partial M^{r}$ at $q$, with principal curvature direction $u_{i}$, and $K_{i j}$ is the sectional curvature of the plane spanned by $u_{i}$ and $u_{j}$. Now $\nabla_{u} \partial_{r}=0$ for horizontal $u$, and $\nabla_{(1 / G) \partial_{\vartheta}} \partial_{r}=G^{-2} G_{r} \partial_{\vartheta}$. Thus

$$
\int_{M^{r}} \chi=\chi(S)-\frac{1}{4 \pi^{2}} \int_{\partial M^{r}} K_{h} G^{-1} G_{r} \omega_{r}
$$

where $K_{h}(q)$ is the sectional curvature of the unique horizontal 2-plane contained in $\left(\partial M^{r}\right)_{q}$. Since the restriction of $\pi$ to $\partial M^{r}$ is a Riemannian submersion, Fubini's theorem yields:

$$
\begin{aligned}
\int_{\partial M^{r}} K_{h} G^{-1} G_{r} \omega_{r} & =\int_{\partial M^{r}}\left\{K_{S}-\frac{3}{4} f^{2} G^{2}\right\} G^{-1} G_{r} \omega_{r} \\
& =\int_{S} K_{S}\left(\int_{0}^{2 \pi} G_{r} d \vartheta\right) \omega_{s}-\frac{3}{4} \int_{S} f^{2}\left(\int_{0}^{2 \pi} G^{2} G_{r} d \vartheta\right) \omega_{s} .
\end{aligned}
$$

Here, $f$ is defined by the equation $\Omega=f \omega_{s}$. Now

$$
\begin{aligned}
\int_{S} f^{2}\left(\int_{0}^{2 \pi} G^{2} G_{r} d \vartheta\right) \omega_{s} & =\int_{\{f \neq 0\}} f^{2}\left(\int_{0}^{2 \pi} G^{2} G_{r} d \vartheta\right) \omega_{s} \\
& \leq \frac{4}{3} \int_{\{f \neq 0\}} K_{S}\left(\int_{0}^{2 \pi} G_{r} d \vartheta\right) \omega_{s}
\end{aligned}
$$

by $1.3(\mathrm{ii})$. Thus

$$
\lim _{r \rightarrow \infty} \int_{S} f^{2}\left(\int_{0}^{2 \pi} G^{2} G_{r} d \vartheta\right) \omega_{s}=0
$$


and

$$
\begin{aligned}
\int_{M} \chi & =\lim _{r \rightarrow \infty} \int_{M^{r}} \chi=\chi(S)-\frac{1}{4 \pi^{2}} \lim _{r \rightarrow \infty} \int_{S} K_{s}\left(\int_{0}^{2 \pi} G_{r} d \vartheta\right) \omega_{s} \\
& =\frac{1}{4 \pi^{2}} \lim _{r \rightarrow \infty} \int_{S}\left(\int_{0}^{2 \pi} 1-G_{r} d \vartheta\right) K_{s} \omega_{s}=\frac{1}{2 \pi} \int_{S} \kappa K_{S} \omega_{s} .
\end{aligned}
$$

The last statement of the theorem now follows from 1.6.

\section{Some metrics on vector bundles over spheres}

Theorem 1.4 shows that the flat bundle case is rigid. The standard examples of nonnegative curvature in the nonflat case are found in [2] and [3]. We briefly recall this construction for fibers diffeomorphic to $\mathbf{R}^{2}$ :

Let $G$ be a Lie group with bi-invariant metric, and let $P_{2}$ denote $\mathbf{R}^{2}$ together with a metric of nonnegative curvature. Suppose $H$ is a closed subgroup of $G$ which acts on $P_{2}$ by isometries. Then $H$ acts freely on the Riemannian product $G \times P_{2}$ via $(g, m) \mapsto\left(g h, h^{-1} m\right)$, and there is a metric of nonnegative curvature on the quotient $M=G \times_{H} P_{2}$ with respect to which the projection $\pi: G \times P_{2} \rightarrow M$ becomes a Riemannian submersion. For example, let $G=S^{3}$, and $H=S^{1}$ acting on $\mathrm{R}^{2}$ by rotations around the origin, so that $M$ is topologically the 2-dimensional vector bundle over $S^{2}$ associated with the Hopf fibration. It is straightforward to check that with the above metric, the soul (= the zero section) of $M$ is isometric to the 2-sphere of constant curvature 4 . The fibers are totally geodesic, and with the notation of $\S 1, G=r /\left(1+r^{2}\right)^{1 / 2}$, while $f \equiv 2$.

In contrast to the rigidity when $\nu(S)$ is flat, one has

Theorem 2.1. Consider $M=S^{3} \times{ }_{S^{1}} \mathbf{R}^{2}$ with the standard submersion metric. Let $h$ denote an arbitrary real valued function with compact support in $M-S$ and with bounded derivatives up to order 2 . Then for small enough $\varepsilon>$ 0 , the metric on $M$ obtained by deforming $G$ to $\tilde{G}=G+\varepsilon h$ has nonnegative sectional curvature.

Notice that if one chooses $h$ so that $h_{\vartheta} \neq 0$, then the resulting metric on $M$ cannot originate from the construction described above, i.e., $M$ is not isometrically a quotient $S^{3} \times{ }_{S^{1}} \mathbf{R}^{2}$ for any metrics on $S^{3}$ and $\mathbf{R}^{2}$, since in such a quotient, $\partial_{\vartheta}$ must be a Killing field, implying $G_{\vartheta}=0$.

Before proceeding to the proof of the theorem, we include for future reference some results that are valid for any 4-dimensional manifold $M$ in the context of 1.2(b). $X, Y$ will denote a local oriented orthonormal basis of vector fields on $S$, as well as their horizontal lifts. $\mu:=(X G) / G$ and $\lambda:=(Y G) / G$ are the principal curvatures of the fibers of $\pi: M \rightarrow S$ in directions $X$ and $Y$ 
respectively. Then straightforward computations yield

$$
\begin{aligned}
\nabla_{X} \partial_{r} & =\nabla_{\partial_{r}} X=0 ; \\
\nabla_{X} \partial_{\vartheta} & =\nabla_{\partial_{\vartheta}} X=\mu \partial_{\vartheta}+\frac{1}{2} f G^{2} Y ; \\
\nabla_{Y} \partial_{\vartheta} & =\nabla_{\partial_{\vartheta}} Y=\lambda \partial_{\vartheta}-\frac{1}{2} f G^{2} X ; \\
\nabla_{\partial_{r}} \partial_{\vartheta} & =\nabla_{\partial_{\vartheta}} \partial_{r}=G^{-1} G_{r} \partial_{\vartheta} ; \\
\nabla_{\partial_{r}} \partial_{r} & =0 ; \\
\nabla_{\partial_{\vartheta}} \partial_{\vartheta} & =G^{-1} G_{\vartheta} \partial_{\vartheta}-G G_{r} \partial_{r}-G^{2} \mu X-G^{2} \lambda Y .
\end{aligned}
$$

These equalities in turn imply

$$
\begin{aligned}
& R\left(X, \partial_{r}\right) \partial_{r}=R\left(\partial_{r}, X\right) X=0 ; \\
& 2 R\left(X, \partial_{r}\right) Y=R(X, Y) \partial_{r}=f G^{-1} G_{r} \partial_{\vartheta} ; \\
& K_{X, \partial_{\vartheta}}=G^{-1}\left(\nabla_{X} X-X X\right) G+\frac{1}{4} f^{2} G^{2} ; \\
& K_{Y, \partial_{\vartheta}}=G^{-1}\left(\nabla_{Y} Y-Y Y\right) G+\frac{1}{4} f^{2} G^{2} ; \\
& K_{\text {fiber }}=-G^{-1} G_{r r} ; \\
& \left\langle R\left(\partial_{\vartheta}, X\right) X, Y\right\rangle=-\frac{1}{2}\left\{(X f) G^{2}+3 f G(X G)\right\} ; \\
& \left\langle R\left(\partial_{\vartheta}, Y\right) Y, X\right\rangle=\frac{1}{2}\left\{(Y f) G^{2}+3 f G(Y G)\right\} ; \\
& \left\langle R\left(\partial_{r}, \partial_{\vartheta}\right) \partial_{\vartheta}, X\right\rangle=-G \partial_{r} X G ; \\
& \left\langle R\left(\partial_{r}, \partial_{\vartheta}\right), \partial_{\vartheta}, Y\right\rangle=-G \partial_{r} Y G ; \\
& \left\langle R\left(X, \partial_{\vartheta}\right) \partial_{\vartheta}, Y\right\rangle=G\left(\nabla_{X} Y-X Y\right) G ; \\
& K_{X, Y}=K_{S}-\frac{3}{4} f^{2} G^{2} .
\end{aligned}
$$

Proof of 2.1. It is not hard to see that the only planes of zero curvature in the $G$-metric are those spanned by $\partial_{r}$ and a horizontal vector. Thus, by choosing $\varepsilon$ small enough, we need only consider expressions of the form $\langle R(U, V) V, U\rangle$, where $U=X+\alpha \partial_{r}+\beta \partial_{\vartheta}, V=\partial_{r}+\gamma X+\delta Y+$ $\varsigma \partial_{\vartheta}, \alpha, \beta, \gamma, \delta, \varsigma \in \mathbf{R}$.

Then

$$
\begin{aligned}
\langle R(U, V) V, U\rangle= & \delta^{2} K_{X, Y}+(\beta \gamma-\varsigma)^{2} \tilde{G}^{2} K_{X, \partial_{\vartheta}}+(\beta \delta)^{2} \tilde{G}^{2} K_{Y, \partial_{\vartheta}} \\
& +(\beta-\alpha \varsigma)^{2} \tilde{G}^{2} K_{\mathrm{fiber}}+2 \delta(\varsigma-\beta \gamma)\left\langle R\left(\partial_{\vartheta}, X\right) X, Y\right\rangle \\
& +3 \delta(\beta-\alpha \varsigma)\left\langle R(X, Y) \partial_{r}, \partial_{\vartheta}\right\rangle \\
& +2(\beta \gamma-\varsigma)(\beta-\alpha \zeta)\left\langle R\left(\partial_{r}, \partial_{\vartheta}\right) \partial_{\vartheta}, X\right\rangle \\
& +2 \beta \delta(\beta-\alpha \zeta)\left\langle R\left(\partial_{r}, \partial_{\vartheta}\right) \partial_{\vartheta}, Y\right\rangle \\
& +2 \beta \delta(\beta \gamma-\varsigma)\left\langle R\left(X, \partial_{\vartheta}\right) \partial_{\vartheta}, Y\right\rangle+2 \beta \delta^{2}\left\langle R\left(\partial_{\vartheta}, Y\right) Y, X\right\rangle
\end{aligned}
$$

Set $x_{1}:=\delta, x_{2}:=\beta-\alpha \varsigma, x_{3}:=\beta \gamma-\varsigma, x_{4}:=\beta \delta$. Then the above expression is a quadratic function of $x=\left(x_{1}, x_{2}, x_{3}, x_{4}\right)$, which by repeated use of $(2.3)$ 
can be written as $Q_{1}(x)+\varepsilon Q_{2}(X)$, where the matrix of $Q_{1}$ is

$$
\left[\begin{array}{cccc}
\left(4+r^{2}\right) /\left(1+r^{2}\right) & 3 r /\left(1+r^{2}\right)^{2} & 0 & 0 \\
3 r /\left(1+r^{2}\right)^{2} & 3 r^{2} /\left(1+r^{2}\right)^{3} & 0 & 0 \\
0 & 0 & r^{4} /\left(1+r^{2}\right)^{2} & 0 \\
0 & 0 & 0 & r^{4} /\left(1+r^{2}\right)^{2}
\end{array}\right]
$$

$Q_{1}$ is positive definite for $r \neq 0$, since the upper left corner matrix has positive trace and determinant. Let $\Theta>0$ be a lower bound for the eigenvalues of $Q_{1}$ on $C:=\operatorname{supp} h$. The hypotheses on $h$ imply that there is an $\eta>0$ such that $\left|Q_{2}(x)\right| \leq \eta\|x\|^{2}$ on $C$ for all $\varepsilon$ say, less than 1 . Choose $0<\varepsilon<$ $\min \{\Theta / \eta, 1\}$. Then $\left(Q_{1}+\varepsilon Q_{2}\right)(x) \geq Q_{1}(x)-\varepsilon\left|Q_{2}(x)\right| \geq 0$. Thus the $\tilde{G}$ metric has nonnegative curvature. Uniform boundedness in $\varepsilon$ is crucial here, and the reader may want to compare this construction with the one given in [1]. q.e.d.

The associated bundle construction in [2] shows that any $\mathbf{R}^{2}$-bundle over $S^{n}$ admits a metric of nonnegative curvature. Actually, a somewhat stronger result is true:

Theorem 2.4. Let $S$ denote the $n$-sphere together with some metric of positive curvature, and let $\pi: E \rightarrow S$ be a 2-dimensional vector bundle over $S$. Then there exists a family of metrics of nonnegative curvature on $E$, each of which has soul isometric to $S$, with totally geodesic fibers.

Proof. For $n>2, E$ is a trivial bundle (cf. [8]), and one then takes the isometric product $S \times P_{2}$, where $P_{2}$ is $\mathbf{R}^{2}$ together with any metric of nonnegative curvature. Assume then that $n=2$ and that $E$ is nontrivial. By the classification theorem of bundles over spheres, every vector bundle over the 2-sphere is orientable (cf. [8]). Choose an orientation of $E$. As before, given a Riemannian connection on $E$ with curvature tensor $R$, the corresponding curvature form $\Omega$ will be identified with $f \omega$ where $\omega$ is the volume form of $S$, and $f: S \rightarrow R$ is given locally by $f=\Omega(X, Y)(U, J U), X, Y$ local oriented orthonormal vector fields on $S, U$ local section of $E$ with $\|U\|=1$.

Fix any Riemannian connection on $E$, and let $\Omega$ denote its curvature form. Set

$$
c:=\frac{1}{\operatorname{vol} S} \int_{S} \Omega=\left(\int_{S} \Omega\right) /\left(\int_{S} \omega\right),
$$

and $\tilde{\Omega}=c \omega . c \neq 0$ since $E$ is nontrivial.

We claim there exists a Riemannian connection $\tilde{\nabla}$ on $E$ with curvature form $=\tilde{\Omega}$. To see this, notice that $\int_{S}(\tilde{\Omega}-\Omega)=0$, so that $\tilde{\Omega}=\Omega+d \Theta$, for some 1-form $\Theta$ on $S$.

Now define $\tilde{\nabla}$ by $\tilde{\nabla}_{X} U=\nabla_{X} U+\Theta(X) J U, X \in \mathfrak{X} S, U \in \Gamma E$, where $J$ is the canonical complex structure on $E$. $J$ is parallel with respect to $\nabla$, and 
it is easily verified that $\tilde{\nabla}$ is a Riemannian connection. If $\tilde{R}$ is the curvature tensor of $\tilde{\nabla}$, then

$$
\begin{aligned}
\tilde{R}(X, Y) U= & \tilde{\nabla}_{X}\left(\nabla_{Y} U+\Theta(Y) J U\right)-\tilde{\nabla}_{Y}\left(\nabla_{X} U+\Theta(X) J U\right)-\tilde{\nabla}_{[X, Y]} U \\
= & R(X, Y) U+\Theta(X) J \nabla_{Y} U+\nabla_{X}(\Theta(Y) J U) \\
& +\Theta(X) \Theta(Y) J U-\Theta(Y) J \nabla_{X} U-\nabla_{Y}(\Theta(X) J U) \\
& -\Theta(Y) \Theta(X) J U-\Theta([X, Y]) J U \\
= & R(X, Y) U+d \Theta(X, Y) J U
\end{aligned}
$$

Thus the curvature form of $\tilde{\nabla}$ is $\tilde{\Omega}$. Now choose a Riemannian connection $\nabla$ as above, so that $\Omega=c \omega$. Given $u \in E$, let $A_{u}$ denote the canonical vector space isomorphism between the fiber through $u$ and its tangent space at $u$. One has the vector fields $\partial_{r}, \partial_{\vartheta}$ on $E-S$ given by

$$
\left.\partial_{r}\right|_{u}=A_{u} u /\|u\|,\left.\quad \partial_{\vartheta}\right|_{u}=A_{u} J u, \quad u \in E-S .
$$

Next define a Riemannian metric on $E$ as follows: $\pi: E \rightarrow S$ is to be a Riemannian submersion, where the horizontal subspaces are those determined by the connection $\nabla$, and the metric on the fibers is taken to be $d r^{2}+G^{2} d \vartheta^{2}$, with $G:=\varepsilon r /\left(\varepsilon^{2}+r^{2}\right)^{1 / 2}$ for some fixed $\varepsilon>0$ satisfying $\varepsilon^{2}<\left(4 / 3 c^{2}\right) \min K_{s}\left(K_{S}=\right.$ sectional curvature of $\left.S\right)$. Notice that replacing the connection $\nabla$ by $\tilde{\nabla}, \tilde{\nabla}_{X} U:=\nabla_{X} U+d h(X) J U$, for $h: S \rightarrow \mathbf{R}$, changes the horizontal distribution and therefore the metric, even though the curvature form remains unchanged. Thus $\Omega=c \omega$ actually determines a family of metrics on $E$. A standard argument shows that (2.2) and (2.3) remain valid, with $G$ as above, $f \equiv c, \mu=\lambda \equiv 0$. In particular, the fibers of $E$ are totally geodesic. To see that $E$ has nonnegative curvature, consider $u, w \in E_{q}$. If $q \in E-S$, then there exist local basic $X, Y,\{X, Y\}$ oriented orthonormal, such that

$u=\left.\left(\eta X+\alpha \partial_{r}+\beta \partial_{\vartheta}\right)\right|_{q}, w=\left.\left(\Theta \partial_{r}+\gamma X+\delta Y+\varsigma \partial_{\vartheta}\right)\right|_{q}, \alpha, \beta, \gamma, \delta, \zeta, \eta, \Theta \in \mathbf{R}$.

Simplifying and grouping terms,

$$
\begin{aligned}
\langle R(u, w) w, u\rangle= & (\eta \delta)^{2} K_{X, Y}+(\beta \Theta-\alpha \zeta)^{2} G^{2} K_{\partial_{r}, \partial_{\vartheta}}+(\beta \gamma-\eta \zeta)^{2} G^{2} K_{X, \partial_{\vartheta}} \\
& +(\beta \delta)^{2} G^{2} K_{Y, \partial_{\vartheta}}+3 \eta \delta(\beta \Theta-\alpha \zeta)\left\langle R(X, Y) \partial_{r}, \partial_{\vartheta}\right\rangle,
\end{aligned}
$$

where the right side is evaluated at $q$.

Thus $\langle R(u, w) w, u\rangle=Q(\eta \delta, \beta \Theta-\alpha \zeta, \beta \gamma-\eta \zeta, \beta \delta)$, where $Q: \mathbf{R}^{4} \rightarrow \mathbf{R}$ is the quadratic function with matrix

$$
A=\left[\begin{array}{cccc}
K_{X, Y} & \frac{3}{2}\left\langle R(X, Y) \partial_{r}, \partial_{\vartheta}\right\rangle & 0 & 0 \\
\frac{3}{2}\left\langle R(X, Y) \partial_{r}, \partial_{\vartheta}\right\rangle & G^{2} K_{\partial_{r}, \partial_{\vartheta}} & 0 & 0 \\
0 & 0 & G^{2} K_{X, \partial_{\vartheta}} & 0 \\
0 & 0 & 0 & G^{2} K_{Y, \partial_{\vartheta}}
\end{array}\right]
$$


Now $K_{X, \partial_{\vartheta}}=K_{Y, \partial_{\vartheta}}=\frac{1}{4} c^{2} G^{2}>0$, so $A$ is positive definite iff its upper left corner

$$
B=\left[\begin{array}{cc}
K_{S}-\frac{3}{4} c^{2} G^{2} & \frac{3}{2} c G G_{r} \\
\frac{3}{2} c G G_{r} & -G G_{r r}
\end{array}\right]
$$

is positive definite. But $K_{S}-\frac{3}{4} c^{2} G^{2}>K_{S}-\frac{3}{4} c^{2} \varepsilon^{2}>0$ by choice of $\varepsilon$, while $-G G_{r r}=3 \varepsilon^{2} G^{2} /\left(\varepsilon^{2}+r^{2}\right)^{2}>0$. Thus the trace of $B$ is positive. Finally,

$$
\begin{aligned}
\frac{\operatorname{det} B}{G^{2}} & =\left(K_{s}-\frac{3}{4} \frac{c^{2} \varepsilon^{2} r^{2}}{\varepsilon^{2}+r^{2}}\right) \cdot \frac{3 \varepsilon^{2}}{\left(\varepsilon^{2}+r^{2}\right)^{2}}-\frac{9}{4} \frac{c^{2} \varepsilon^{6}}{\left(\varepsilon^{2}+r^{2}\right)^{3}} \\
& =3 \varepsilon^{2}\left(K_{S}-\frac{3}{4} c^{2} \varepsilon^{2}\right) /\left(\varepsilon^{2}+r^{2}\right)^{2}>0 .
\end{aligned}
$$

Therefore $B$ is positive definite, and $\langle R(u, w) w, u\rangle \geq 0$. It is worth mentioning that the only nontrivial solutions for $\langle R(u, w) w, u\rangle=0$ are $\operatorname{span}\{u, w\}=$ span $\left\{\right.$ horizontal vector, $\left.\partial_{r}\right\}$.

When $q \in S$, one replaces $\partial_{r}, \partial_{\vartheta}$ by an orthonormal basis of $S_{q}^{\perp}$. The matrix $A$ then becomes

$$
\left[\begin{array}{cccc}
K_{S} & \frac{3}{2} c & 0 & 0 \\
\frac{3}{2} c & 3 \varepsilon^{-2} & 0 & 0 \\
0 & 0 & 0 & 0 \\
0 & 0 & 0 & 0
\end{array}\right]
$$

which again is nonnegative.

\section{References}

[1] J.-P. Bourguignon, A. Deschamps \& P. Sentenac, Quelques variations particulières d'un produit de métriques, Ann. Sci. École Norm. Sup. (4) 6 (1973) 1-16.

[2] J. Cheeger, Some examples of manifolds of nonnegative curvature, J. Differential Geometry 8 (1972) 623-628.

[3] J. Cheeger \& D. Gromoll, On the structure of complete manifolds of nonnegative curvature, Ann. of Math. 96 (1972) 413-443.

[4] S. Cohn-Vossen, Kürzeste Wege und Totalkrümmung auf Flächen, Composito Math. 2 (1935) 69-133.

[5] R. Hermann, A sufficient condition that a map of Riemannian manifolds be a fiber bundle, Proc. Amer. Math. Soc. 11 (1960) 236-242.

[6] B. O'Neill, The fundamental equations of a submersion, Michigan Math. J. 13 (1966) 459-469.

[7] W. Poor, Some results on nonnegatively curved manifolds, J. Differential Geometry 9 (1974) 583-600.

[8] N. Steenrod, The topology of fiber bundles, Princeton University Press, Princeton, NJ, 1951.

[9] J. Vilms, Totally geodesic maps, J. Differential Geometry 4 (1970) 73-79.

State UNIVERSity OF NEW YORK, STONY BROOK 
